\title{
PERIOPERATIVE ANALGESIA FOR SHOULDER ARTHROPLASTY: A CRITICAL REVIEW OF CURRENT PRACTICES IN A TERTIARY HOSPITAL
}

\author{
A. Hassan, N. Bedforth
}

Department of Anaesthesia, Nottingham University Hospitals NHS Trust, Nottingham, UK

\section{Introduction:}

Shoulder Arthroplasty (SA) can cause severe postoperative pain, but consensus on the optimal analgesic approach is lacking. Continuous catheter-based Interscalene block (CISB) prolongs the analgesic benefits of its single injection counterpart (SISB), but there are concerns over CISB complications. The aim of this review was to evaluate postoperative analgesia and side effects of CISB compared with SISB for SA.

\section{Methods:}

A retrospective review of 60 SA patients; random selection of equal numbers receiving general anaesthesia plus either CISB or SISB. Average postoperative pain for days zero, one and two were assessed with a verbal categorical rating scale (VRS). Postoperative opioid requirements were determined and converted to equal doses of intravenous morphine (converted to equivalent doses of intravenous morphine). Duration of hospital stay, postoperative oxygen administration and hypoxemia episodes (arterial oxygen saturation $<92 \%$ in non COPD patients ; $<88 \%$ in COPD patients) were collected from the electronic health records.

\begin{tabular}{|c|c|c|}
\hline & $\begin{array}{l}\text { Continuous catheter- } \\
\text { based Interscalene } \\
\text { block } \\
\text { CISB } \\
30 \text { Patients }\end{array}$ & $\begin{array}{c}\text { Single injection } \\
\text { Interscalene } \\
\text { block } \\
\text { SISB } \\
30 \text { Patients }\end{array}$ \\
\hline Age Distribution (Median) & 74 & 74 \\
\hline Age Distribution (Range) & $47: 82$ years & $49: 86$ years \\
\hline Sex Distribution & 25 Females / 5 Males & 19 Females / 11 Males \\
\hline \multicolumn{3}{|l|}{ Comorbidities } \\
\hline Diabetes & 1 & 7 \\
\hline CHRONIC PAIN & 7 & 4 \\
\hline COPD & 5 & 2 \\
\hline OSA & 2 & 3 \\
\hline ASTHMA & 5 & 5 \\
\hline CKD & 2 & 4 \\
\hline \multicolumn{3}{|l|}{ Type of surgery } \\
\hline Primary & $22(73 \%)$ & $23(76 \%)$ \\
\hline Revision & $8(27 \%)$ & $7(23 \%)$ \\
\hline
\end{tabular}

Table 1: Patients demographics and type of surgery in both groups

\section{Results}

We reviewed 60 patients who underwent SA from September 2017 until December 2018. Demographic and clinical characteristics of patients in both groups were nearly equivalent. Pain scores were similar with no patients complaining of severe pain in either group and the majority of patients scoring no pain or mild pain throughout. Opioid requirements were modest throughout. 57\% of patients in CISB group required postoperative oxygen compared with only $10 \%$ in SISB group. $20 \%$ of patients in CISB group had hypoxaemia episodes compared with $10 \%$ in SISB group. More than $40 \%$ of patients in both groups stayed in the hospital 3 days or more postoperatively.

\begin{tabular}{|c|c|c|}
\hline & CISB & SISB \\
\hline Day 0 (day of the operation) & 30 patients & 30 patients \\
\hline No pain & $22(73 \%)$ & $23(77 \%)$ \\
\hline mild & $5(17 \%)$ & $7(23 \%)$ \\
\hline moderate & $3(10 \%)$ & - \\
\hline severe & - & - \\
\hline $\begin{array}{l}\text { Equivalent median morphine } \\
\text { dose; mg (IQR) }\end{array}$ & $3.3(0-3.7)$ & $5(4.6-5.4)$ \\
\hline Day 1 & 30 patients & 30 patients \\
\hline No pain & $9(30 \%)$ & $8(27 \%)$ \\
\hline mild & $19(63 \%)$ & $21(70 \%)$ \\
\hline moderate & $2(7 \%)$ & $1(3 \%)$ \\
\hline severe & - & - \\
\hline $\begin{array}{l}\text { Equivalent median morphine } \\
\text { dose; mg (IQR) }\end{array}$ & $7(5-16.6)$ & $14.5(10-20.9)$ \\
\hline Day 2 & 25 patients & 26 patients \\
\hline No pain & $6(24 \%)$ & $8(31 \%)$ \\
\hline Mild & $13(52 \%)$ & $13(50 \%)$ \\
\hline moderate & $6(24 \%)$ & $5(19 \%)$ \\
\hline severe & - & - \\
\hline $\begin{array}{l}\text { Equivalent median morphine } \\
\text { dose; mg (IQR) }\end{array}$ & $10(2.5-16.6)$ & $6(5-13.9)$ \\
\hline
\end{tabular}

Table2 : Mean post-operative pain scores \& median opioid requirements following SA

\begin{tabular}{|c|c|c|}
\hline & CISB & SISB \\
\hline 1 Day & $6(20 \%)$ & $4 \quad(13 \%)$ \\
\hline 2 Days & 11 (37\%) & $12(40 \%)$ \\
\hline$\geq 3$ days & 13 (43\%) & $14(47 \%)$ \\
\hline $\begin{array}{l}\text { Recorded reasons } \\
\text { for late discharge (if } \\
\geq 3 \text { days) }\end{array}$ & $\begin{array}{l}\text { - } \text { AKI } \\
\text { - Pain Issues } \\
\text { - } \text { CO2 retention } \\
\text { - } \text { Basal Atelectasis } \\
\text { - } \text { SOB } \\
\text { - Chest infection }\end{array}$ & $\begin{array}{ll}: & \text { AF } \\
: & \text { Pain Issues } \\
\text { Hyponatraemia }\end{array}$ \\
\hline
\end{tabular}

\section{Conclusions:}

CISB and SISB produced satisfactory analgesia. Patients receiving CISB had a higher incidence of hypoxaemia and requirement for oxygen therapy. Application of enhanced recovery in Shoulder Arthroplasty is ongoing to decrease the length of postoperative stay and unify the practice.

\section{References}

L. Vorobeichik1, R. Brull. Should continuous rather than single-injection interscalene block be routinely offered for major shoulder surgery? A meta-analysis of the analgesic and side-effects profiles. British Journal of Anaesthesia, 120 (4): 679e692 (2018). 